\title{
Evaluation of reverse transcription-loop-mediated isothermal amplification (RT-LAMP) for detecting SARS-CoV-2 in clinical, environmental and animal samples.
}

Fatiha M. Benslimane*, Ola Al-Jamal, Sonia Boghattas, Asmaa A. Althani, Hadi M. Yassine Biomedical Research Center, Qatar University, PO Box 2713, Doha, QATAR

*Corresponding Author

\section{BACKGROUND}

The current SARS-CoV-2 pandemic has created an unprecedented global demand for rapid diagnostic testing. Most countries are employing reverse transcriptase quantitative PCR (RT-qPCR) for confirmation of infection. Consequently, a global shortage of RNA extraction kits as well as RT-qPCR assay kits and their associated reagents has ensued. Therefore, alternative diagnostics not dependent on these commonly used materials are required. First described 20 years ago by Notomi et al., the loop-mediated isothermal amplification (LAMP) assay is robust, rapid and straightforward, yet retains high sensitivity and specificity.

These features have seen the LAMP assay and the inclusion of a reverse transcriptase (RT-LAMP) implemented for a broad range of molecular diagnostic applications extending from infectious diseases, including detection of the original SARS-CoV-1 virus.

The advantages of RT-LAMP include using different reagents than RT-qPCR, the potential for direct processing of samples without the need for prior RNA extraction and an extremely rapid turnaround time. Several groups have now described different RTLAMP assays for detection of SARS-CoV-2 RNA.

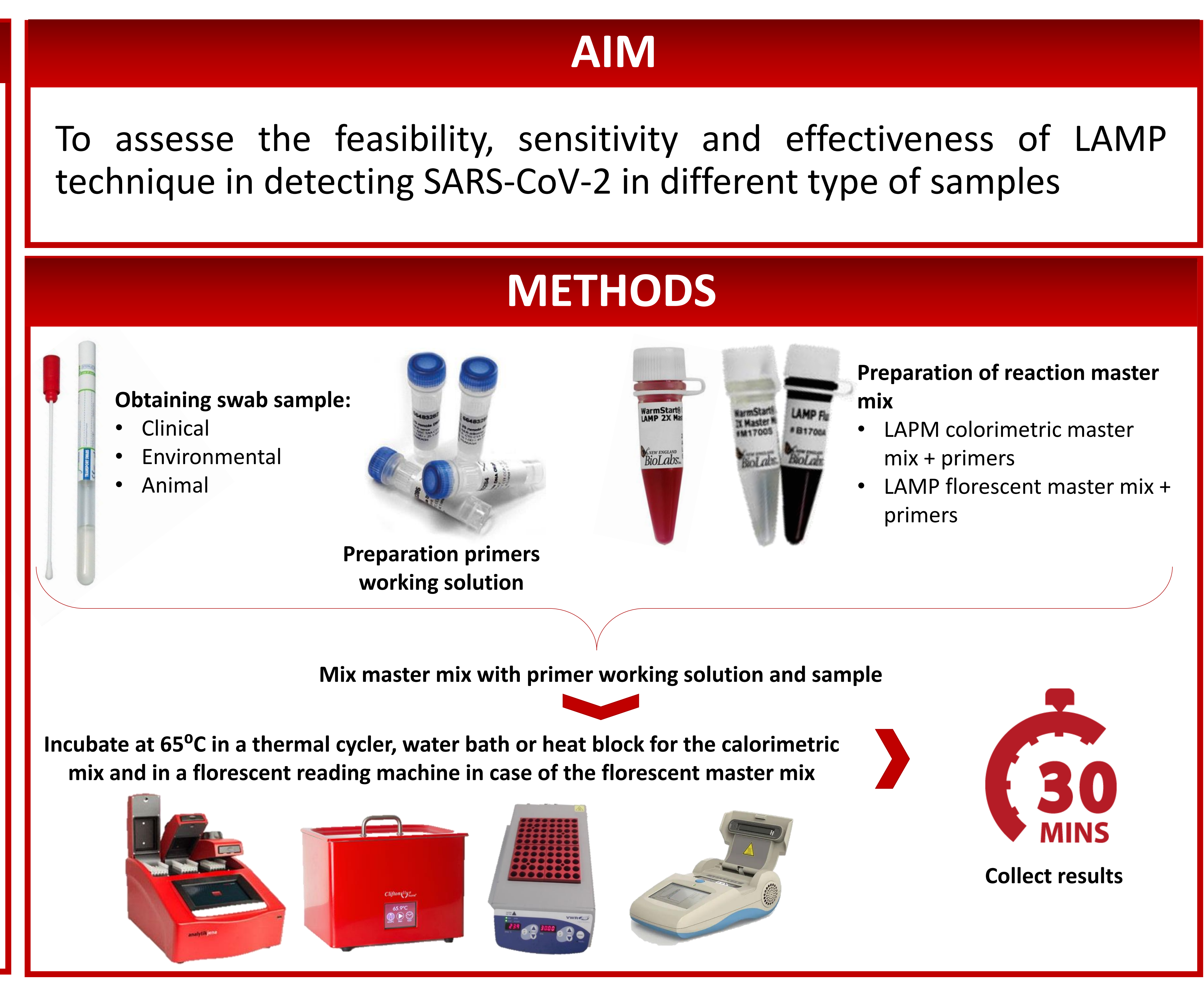

\section{RESULTS}

Lamp sensitivity testing using SARS-CoV-2 control
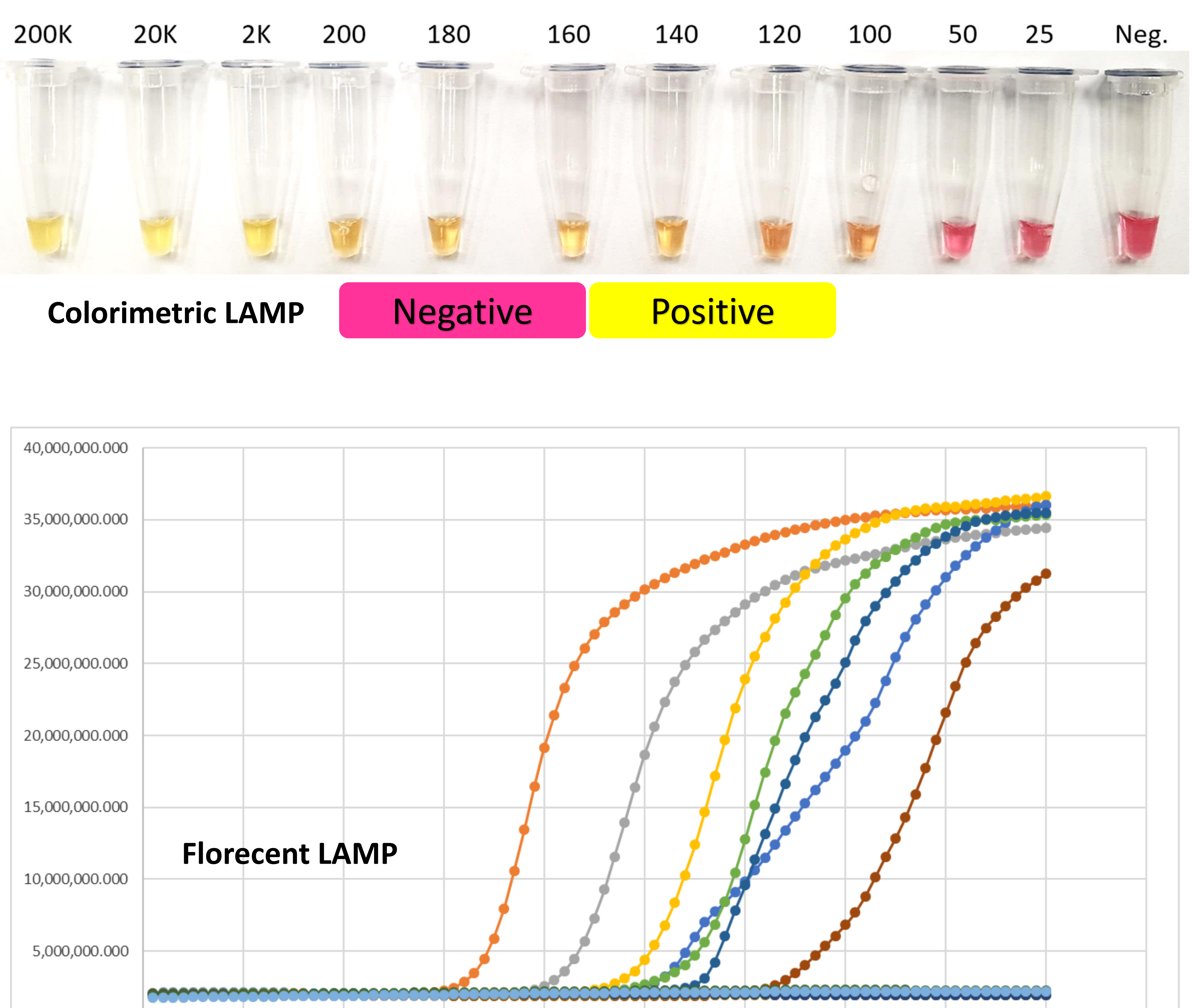

No extraction, testing sample of different $\mathrm{CT}$ values

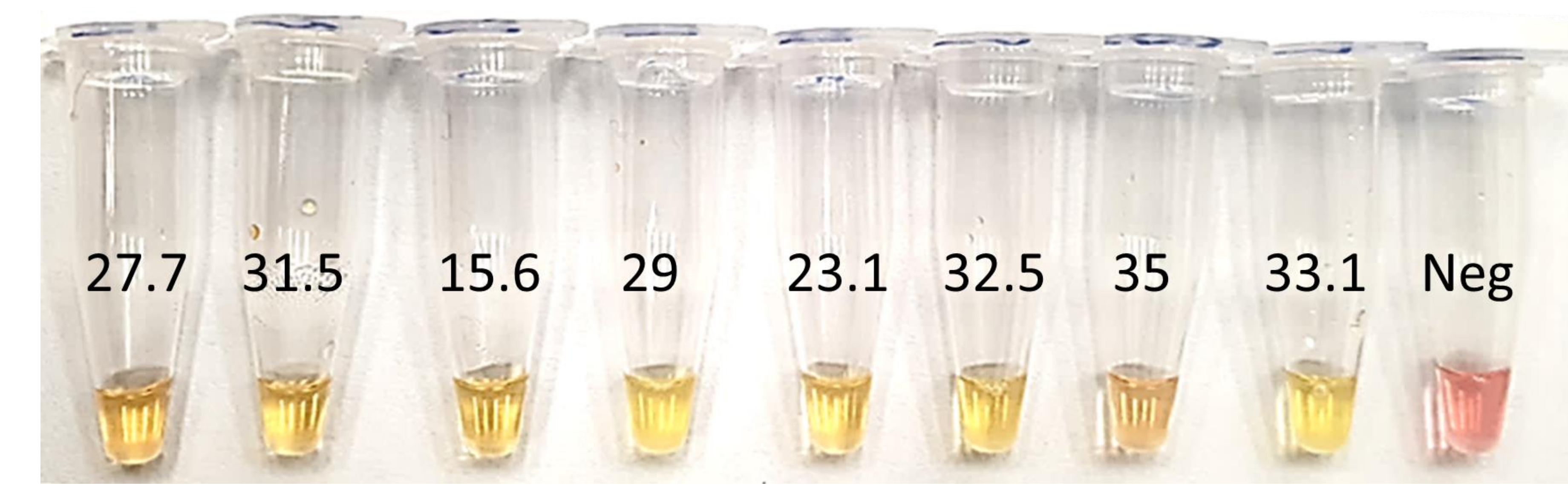

Samples of CT 20.18 serially diluted

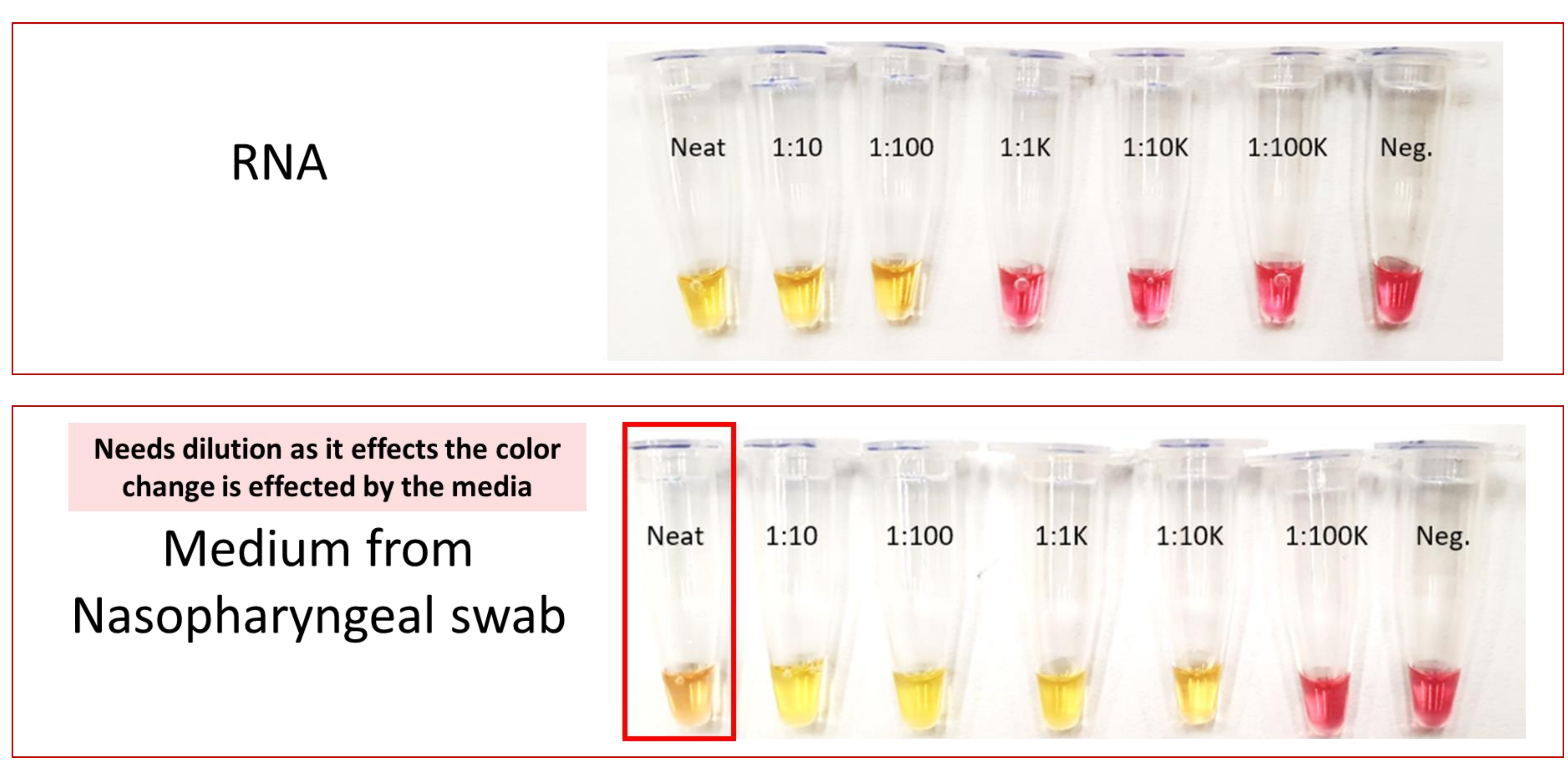

\section{CONCLUSION}

In just 30 min we were able to detect the virus with out any prior sample processing. Our primers were able to detecte up to 100 copies of the viruses, which is comparable to the RT-PCR that we currently use in our lab. The primers were tested against all other coronavirus and they have shown $100 \%$ specificity to the novel SARS-CoV-2 virus. Both the florescent and calorimetric master mixes were able to detect the virus in all tested samples: clinical, animal and environmental. In conclusion, LAMP is a fast reliable technique that could be used as a quick screening method for the detection of SARSCoV-2 in different settings and using different collection medium.
ACKNOWLEDGEMENT

This work was supported by Qatar Petroleum under Qatar University external grant (QUEX-BRC-WP-18/19)

We acknowledge the support from the Ministry of Public Health, Doha, Qatar and the virology laboratory in Hamad Medical Corporation, Doha, Qatar for their efforts in the preparation of nasopharyngeal samples and collection of clinical data. We thank Dr. Andrew M. Jeremijenko, HMC and the staff of QBB for the collection of environmental sample. 